\title{
A scientific note on Nosema bombi infection intensity among different castes within a Bombus auricomus nest
}

\author{
Chia-Ching $\mathrm{CHU}^{1,2}$, Sydney A. CAMERON ${ }^{3}$ \\ ${ }^{1}$ Department of Crop Sciences, University of Illinois, Urbana, IL, USA \\ ${ }^{2}$ Citrus Research and Education Center, University of Florida, Lake Alfred, FL, USA \\ ${ }^{3}$ Department of Entomology, University of Illinois, 505 S. Goodwin Avenue, Urbana, IL 61801-3795, USA
}

Received 14 December 2015 - Revised 16 May 2016 - Accepted 15 June 2016

parasite / insect caste / bumble bees

Bumble bees (Bombus) provide vital services to agricultural and natural plant systems (Kremen et al. 2002; Fontaine et al. 2006). A survey across diverse North American Bombus species detected higher prevalence of infection by the microsporidian parasite Nosema bombi in declining relative to non-declining species, indicating a correlation between Nosema and Bombus population declines (Cameron et al. 2011). Although it has been shown in the laboratory that $N$. bombi infection is detrimental to the fitness of Bombus terrestris and Bombus lucorum (Otti and Schmid-Hempel 2007; Rutrecht and Brown 2009), much remains unclear regarding N. bombi's ecology and how it interacts with Bombus in the field.

Previous field studies have suggested that Nosema is more prevalent or abundant in males than in females of some Bombus species (Shykoff and Schmid-Hempel 1991; Gillespie 2010; Huth-Schwarz et al. 2012). In a laboratory study, however, contrasting results were found (Rutrecht and Brown 2008). It is possible that genetic differences among Bombus species/Nosema strains, geographic distribution, variability in timing of infection, and within-colony epidemiology (Rutrecht and Brown 2008) may lead to different host-parasite interactions and Nosema infection patterns. Given the association between high Nosema prevalence and specific Bombus taxa

Corresponding author: S. Cameron, scameron@life.illinois.edu Manuscript Editor: David Tarpy undergoing population declines in North America, it is important to examine the host-parasite interactions in other, less-characterized, North American Bombus species.

In a life history study of a $B$. auricomus colony found in Urbana, Illinois (Dean et al. 2015), we detected the presence of $N$. bombi in all of a few individuals tested $(n=10)$. Because these included a sample of bees from each caste (parent and offspring queens (gynes), workers, and males), the colony provided an opportunity for further testing of caste effects on Nosema infection intensity in B. auricomus while minimizing influences of genetic and environmental variation. Here, we examined whether caste effect exists in B. auricomus by comparing Nosema infection intensities in 22 individuals, including the founding queen, seven gynes, seven workers, and seven males.

We extracted gut DNA from the bees using previously described methods (Dean et al. 2015). Initial PCR testing using $N$. bombi-specific primers (NbombiSSUJf1 and NbombiSSUJr1) (Klee et al. 2006) with 10 ng DNA templates found that all samples were infected with $N$. bombi. Initially, male samples appeared to exhibit greater amplicon intensity than those of other castes (Figure 1a). To validate this finding, we conducted qPCR on the samples using $N$. bombi-specific primers (BOMBICAR) (Plischuk et al. 2009), as well as primers targeting a Bombus arginine kinase (AK) gene fragment (Barribeau and Schmid-Hempel 2013). qPCR was conducted using the GoTaq qPCR Master Mix (Promega, Madison, WI) and a 7900HT Fast Real-Time PCR System (Applied Biosystems, Foster City, CA), using standard conditions (annealing at $58^{\circ} \mathrm{C}$ ). Three technical 
a
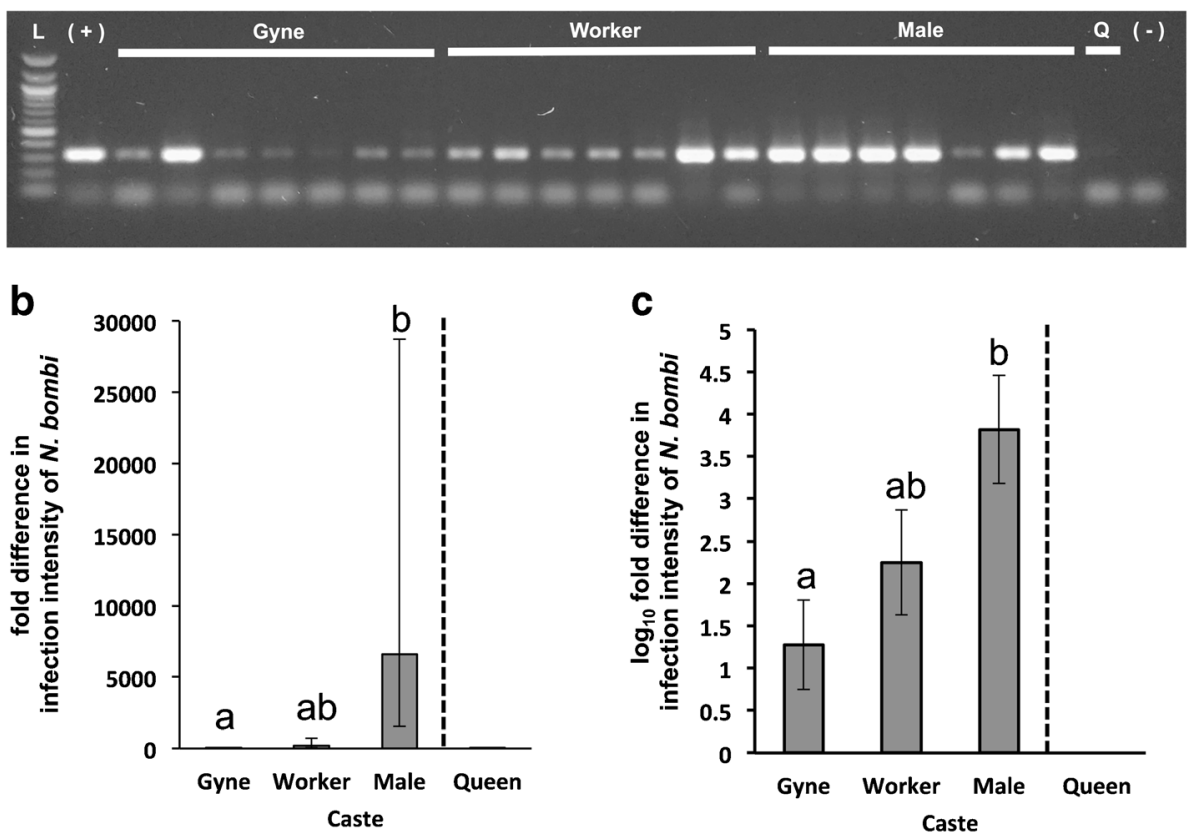

Figure 1. Nosema infection in different $B$. auricomus castes. a Diagnostic PCR detection of $N$. bombi. $L, 100$-bp ladder; (+), positive control, a verified $N$. bombi -infected B. auricomus worker; $Q$, parent queen; (-), negative control. b, c qPCR results showing different $N$. bombi infection intensity in different castes. The data were transformed to fold (b) or $\log _{10}$ fold (c) difference in infection intensity for figure illustration. Different letters indicate significant differences.

replicates were tested for all samples (24 ng DNA template). The PCR efficiencies were 90-100\% $\left(R^{2}>0.99\right)$ and melting curves showed that only the correct amplicons were produced. The data were analyzed using the $2^{-\Delta \Delta C T}$ method (Livak and Schmittgen 2001). The $N$. bombi abundance $\left(\mathrm{C}_{\mathrm{T}}\right)$ was normalized against the Bombus AK gene (to obtain $\Delta \mathrm{C}_{\mathrm{T}}$ ). To correct for haploid/diploid difference between sexes, we halved the infection intensity in male samples by adding 1 to their $\Delta \mathrm{C}_{\mathrm{T}}$ values. For each sample, the intensity $\left(\Delta \mathrm{C}_{\mathrm{T}}\right)$ relative to that of the queen was calculated $\left(\Delta \Delta \mathrm{C}_{\mathrm{T}}\right)$. The $\Delta \Delta \mathrm{C}_{\mathrm{T}}$ values for the workers, gynes, and males were compared using non-parametric Kruskal-Wallis and post hoc tests (NPTESTS; SPSS, IBM, Armonk, NY).

The results indicate that $N$. bombi infection intensity was caste-dependent in this colony of $B$. auricomus $(P=0.027)$. Males had greater $N$. bombi levels than gynes $(P=0.023$, Bonferroni adjusted), while the workers were intermediate between these two castes (Figure $1 \mathrm{~b}, \mathrm{c}$ ). The queen had relatively low $N$. bombi load, similar to that of the gynes (Figure 1b, c). Although it is unclear during which stage of the colony lifecycle the bees were infected, and whether there is an age effect on the data, the fact that both older (queen and worker) and younger (gyne) females had lower Nosema levels than males supports the hypothesis that sex difference is a factor affecting infection intensity.

Caste difference in insects can lead to different physiology, behavior, and gene regulation (Watson et al. 1985; Colgan et al. 2011). Genome ploidy could also influence gene expression or affect pathogen/parasite recognition (Nuismer and Otto 2004; Borges et al. 2012); it is possible that ploidy difference can lead to different susceptibility to Nosema across B. auricomus sexes/castes. The idea that haploid males may be more susceptible to parasite infection than females (O'Donnell and Beshers 2004) has also been tested in some Bombus species using Crithidia and N. bombi (Ruiz-Gonzalez and Brown 2006; Rutrecht and Brown 2008). In these studies, males and females exhibited no significant difference in infection level. It could be that the withincolony epidemiology of Nosema varies with the infection method used (artificial/natural) or is species-dependent, and thus our samples exhibited different infection patterns due to any of these factors. Further investigation is needed to validate the ploidy difference hypothesis.

Bombus males can transmit Nosema during mating (Rutrecht and Brown 2009). Males usually emerge late in the colony life cycle and leave soon afterward as they 
function mostly to mate with gynes outside of the nest; they do not typically return to the colony. Females, on the other hand, comprise the initial worker force and remain in the nest after males have departed, engaging in foraging and the nursing of larvae (Goulson 2003). Females thus have a greater chance of making direct contact with the potentially more susceptible larvae (Van den Eijnde and Vette 1993; Rutrecht and Brown 2008). The gynes are also crucial to colony establishment and may be subject to additional protection (Cremer et al. 2007). Thus, selection for defense against Nosema infection may be stronger in females than in males. From the parasite's perspective, the male's early departure from the colony and higher Nosema load may facilitate the parasite's dissemination. These hypotheses require further testing. Future examination of similar interactions in other Bombus species may help develop approaches contributing to conservation of pollinators in the USA.

\section{ACKNOWLEDGMENTS}

We thank J. B. Whitfield, M. A. Duennes, and C. A. E. Dean for assistance in insect sampling. This work was supported by a U.S. Department of Agriculture, National Institute of Food and Agriculture grant (\#2010-65104-05992).

Note scientifique sur l'intensité de l'infection par Nosema bombi parmi les différentes castes à l'intérieur d'un nid de Bombus auricomus

Eine wissenschaftliche Notiz zur Infektionsintensität von Nosema bombi zwischen verschiedenen Kasten eines Bombus auricomus Nestes

\section{REFERENCES}

Barribeau, S.M., Schmid-Hempel, P. (2013) Qualitatively different immune response of the bumblebee host, Bombus terrestris, to infection by different genotypes of the trypanosome gut parasite, Crithidia bombi . Infect. Genet. Evol. 20, 249-256

Borges, A.A., Humann, F.C., Tavares, M.G., Campos, L.A.O., Hartfelder, K. (2012) Gene copy number and differential gene expression in haploid and diploid males of the stingless bee, Melipona quadrifasciata. Insectes Soc. 59, 587-598

Cameron, S.A., Lozier, J.D., Strange, J.P., Koch, J.B., Cordes, N., et al. (2011) Patterns of widespread decline in North American bumble bees. Proc. Natl. Acad. Sci. USA 108 (2), 662-667

Colgan, T.J., Carolan, J.C., Bridgett, S.J., Sumner, S., Blaxter, M.L., et al. (2011) Polyphenism in social insects: insights from a transcriptome-wide analysis of gene expression in the life stages of the key pollinator, Bombus terrestris . BMC Genomics $12(1), 623$
Cremer, S., Armitage, S.A., Schmid-Hempel, P. (2007) Social immunity. Curr. Biol. 17 (16), R693-702

Dean, C.A.E., Duennes, M.A., Chu, C., Cameron, S.A. (2015) Colony Characteristics and Nosema Infection of Bombus auricomus (Hymenoptera: Apidae). Entomol. News 125(3), 220-223

Fontaine, C., Dajoz, I., Meriguet, J., Loreau, M. (2006) Functional diversity of plant-pollinator interaction webs enhances the persistence of plant communities. PLoS Biol. 4 (1), 129-135

Gillespie, S. (2010) Factors affecting parasite prevalence among wild bumblebees. Ecol. Entomol. 35(6), 737-747

Goulson, D. (2003) Bumblebees, their behaviour and ecology. Oxford University Press, Oxford

Huth-Schwarz, A., Settele, J., Moritz, R.F., Kraus, F.B. (2012) Factors influencing Nosema bombi infections in natural populations of Bombus terrestris (Hymenoptera: Apidae). J. Invertebr. Pathol. 110(1), 48-53

Klee, J., Tek Tay, W., Paxton, R.J. (2006) Specific and sensitive detection of Nosema bombi (Microsporidia: Nosematidae) in bumble bees (Bombus spp.; Hymenoptera: Apidae) by PCR of partial rRNA gene sequences. J. Invertebr. Pathol. 91 (2), 98-104

Kremen, C., Williams, N.M., Thorp, R.W. (2002) Crop pollination from native bees at risk from agricultural intensification. Proc. Natl. Acad. Sci. USA 99(26), 16812-16816

Livak, K.J., Schmittgen, T.D. (2001) Analysis of relative gene expression data using real-time quantitative PCR and the $2^{-}$ $\triangle \Delta \mathrm{CT}$ method. Methods 25, 402-408

Nuismer, S.L., Otto, S.P. (2004) Host-parasite interactions and the evolution of ploidy. Proc. Natl. Acad. Sci. USA 101(30), 11036-11039

O'Donnell, S., Beshers, S.N. (2004) The role of male disease susceptibility in the evolution of haplodiploid insect societies. Proc. R. Soc. Lond. B Biol. Sci. 271 (1542), 979-983

Otti, O., Schmid-Hempel, P. (2007) Nosema bombi: A pollinator parasite with detrimental fitness effects. J. Invertebr. Pathol. $96(2), 118-124$

Plischuk, S., Martin-Hernandez, R., Prieto, L., Lucia, M., Botias, C., et al. (2009) South American native bumblebees (Hymenoptera: Apidae) infected by Nosema ceranae (Microsporidia), an emerging pathogen of honeybees (Apis mellifera ). Environ. Microbiol. Rep. 1 (2), 131-135

Ruiz-Gonzalez, M.X., Brown, M.J.F. (2006) Males vs workers: testing the assumptions of the haploid susceptibility hypothesis in bumblebees. Behav. Ecol. Sociobiol. 60 (4), 501-509

Rutrecht, S.T., Brown, M.J.F. (2008) Within colony dynamics of Nosema bombi infections: disease establishment, epidemiology and potential vertical transmission. Apidologie 39 (5), 504-514

Rutrecht, S.T., Brown, M.J.F. (2009) Differential virulence in a multiple-host parasite of bumble bees: resolving the paradox of parasite survival. Oikos 118, 941-949

Shykoff, J.A., Schmid-Hempel, P. (1991) Incidence and effects of four parasites in natural populations of bumble bees in Switzerland. Apidologie 22 (2), 117-125

Van den Eijnde, J., Vette, N. (1993) Nosema infection in honey bees (Apis mellifera L.) and bumble bees (Bombus terrestris L.). Proc. Sect. Exp. Appl. Entomol. Neth. Entomol. Soc. 4, 205-208

Watson, J.A.L., Okot-Kotber, B.M., Noirot, C. (1985) Caste differentiation in social insects. Pergamon Press Ltd., Oxford 